Submission ID: 43873

\title{
Evaluation Test of Initial Information and Results of Data Processing of Geophysical Surveys of Wells at Creation of Seismogeological Models
}

E.A. Cherepanov* (LUKOIL-Engineering Limited KogalymNIPIneft Branch in Tyumen)

\section{SUMMARY}

In Western Siberia at the heart of the solution of explorative tasks on naphtha and gas the complex of the GIS methods - seismic exploration lies. Seismogeological models are a basis for creation of geological models of oil and gas objects. In recent years, in connection with complication of prospecting tasks, limitation of resources, special attention is paid to increase in effectiveness of geophysical surveys. Increase in requirements to quality of seismogeological models, causes increased requirements to quality of geophysical surveys in the wells providing a petrophysical basis of interpretation. Accuracy of a binding of data of seismic exploration and results of seismogeological constructions in general depends on their quality. In work the adaptive (operated) approach to data processing of the geophysical surveys of wells (GSW) for creation of seismogeological models based on formalization of an index of quality of results of processing and the interconnected accounting of major factors not of the bound to the studied objects is offered. 


\title{
Оценка качества исходной информации и результатов обработки данных ГИС при построении сейсмогеологических моделей
}

\author{
Е.А. Черепанов* (Филиал ООО «ЛУКОЙЛ-Инжиниринг» «КогалымНИПИнефть» в г.Тюмени, \\ ТИУ)
}

\section{Введение}

Сейсмогеологические модели перспективных на нефть и газ объектов являются результатом комплексирования ГИС - сейсморазведка. Очень важным этапом построения сейсмологической модели является этап обработки данных ГИС, направленный на повышение качества данных ГИС за счет устранения (учета) факторов, не связанных с изучаемыми геологическими объектами.

В работе предлагается адаптивный (управляемый) подход к обработке данных геофизических исследований скважин (ГИС) для целей построения сейсмогеологических моделей, основанный на формализации показателя качества результатов обработки и взаимоувязанном учете основных факторов, не связанных с изучаемыми объектами.

\section{Метод}

В Западной Сибири основными факторами негеологического плана, влияющими на качество кривых, являются: технологическое состояние ствола скважины (наличие каверн), аппаратурные ошибки регистрации данных, несоблюдение масштабов записи при регистрации кривых.

С целью оценки качества данных ГИС введено понятие интегральной оценки качества кривых (данных ГИС, используемых для целей построения сейсмогеологических моделей), для этого по интервалу обработки предлагается показатель качества - КК, количественно учитывающий перечисленные выше факторы [1].

КК=(1-Ркав) $\cdot(1-\mathrm{Pa}) \cdot(1-\mathrm{PH})$,

где КК - коэффициент качества, Ркав - вероятность ошибки из-за каверн, Ра - вероятность ошибки из-за недоучета аппаратурных ошибок, Рн - вероятность ошибки, связанной со стандартизацией кривых.

Вероятность ошибок из-за влияния каверн оценивается путем отнесения суммы всех интервалов, осложнённых кавернами к интервалу обработки, и описывается соотношением:

Pкав $=\frac{\sum h \mathrm{kagi}}{\mathrm{H}}$,

где Н - мощность интервала обработки, Гһкаві - сумма толщин каверн в интервале обработки.

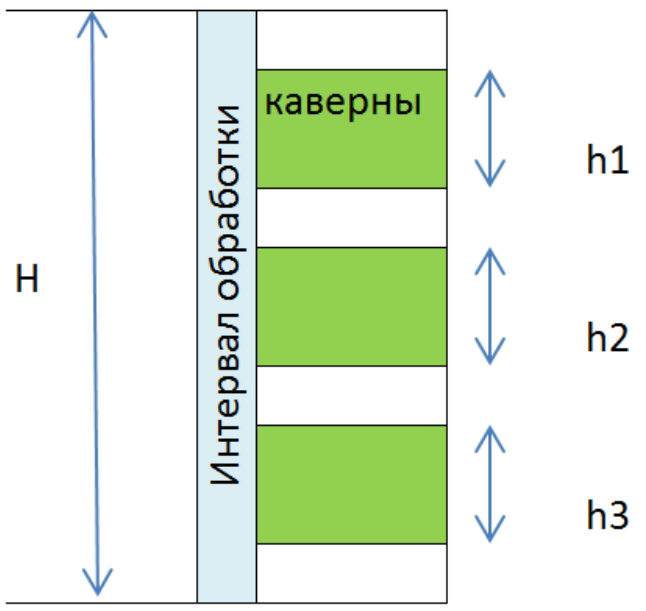

Рисунок 1 Схема анализа вероятности ошибок связанных с влиянием каверн на кривые АК и ГГК-П 


\section{EAGE}

Если все процедуры по исправлению каверн выполнены, то вероятность ошибки Ркав будет стремиться к нулю.

Аналогичным образом, путем отнесения суммы всех интервалов, в которых выявлены аппаратурные ошибки к интервалу обработки, оцениваются срывы записи на каротажных кривых АК и ГГК-П. Сам показатель ${ }^{P a}$ описывается уравнением:

$\mathrm{Pa}=\frac{\sum h \mathrm{ai}}{\mathrm{H}}$

где $\mathrm{H}$ - мощность интервала обработки, इhai - сумма толщин интервалов с выявленными искажениями на записи кривых в результате аппаратурных ошибок.

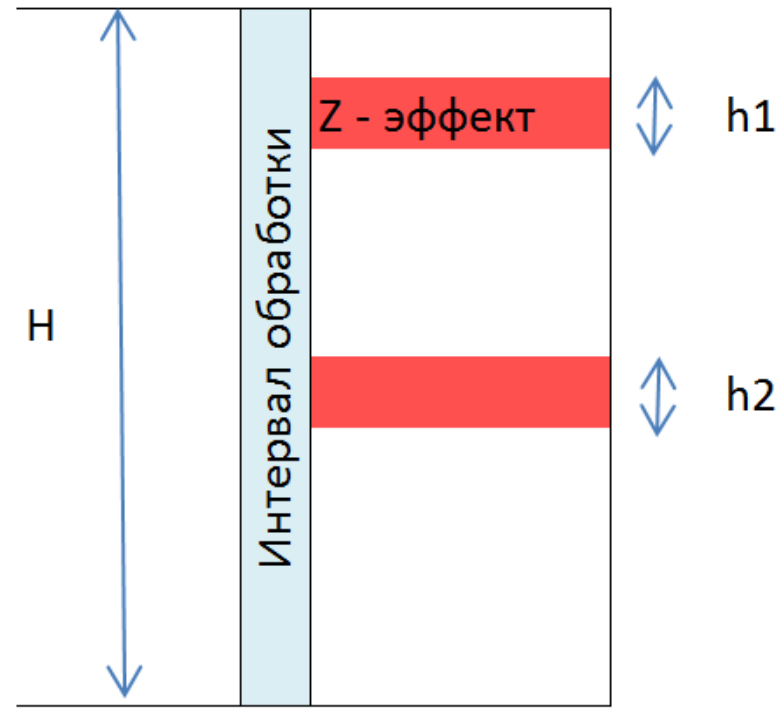

Рисунок 2 Схема анализа вероятности аппаратурных ошибок

При стандартизации кривых АК и ГГК-П проводится их приведение к эталонным величинам опорной скважины. Степень сходимости стандартизируемой и опорной скважин характеризуется коэффициентом корреляции - Raк(ггк- п) между значениями АК (ГГК-П) в текущей и опорной скважинах, который и используется для оценки вероятности ошибки, связанной с нормализацией (масштабированием) кривых.

$\mathrm{P}_{\mathrm{H}}=1-\operatorname{Ra\kappa }(\mathrm{rг \kappa}-\Pi)$

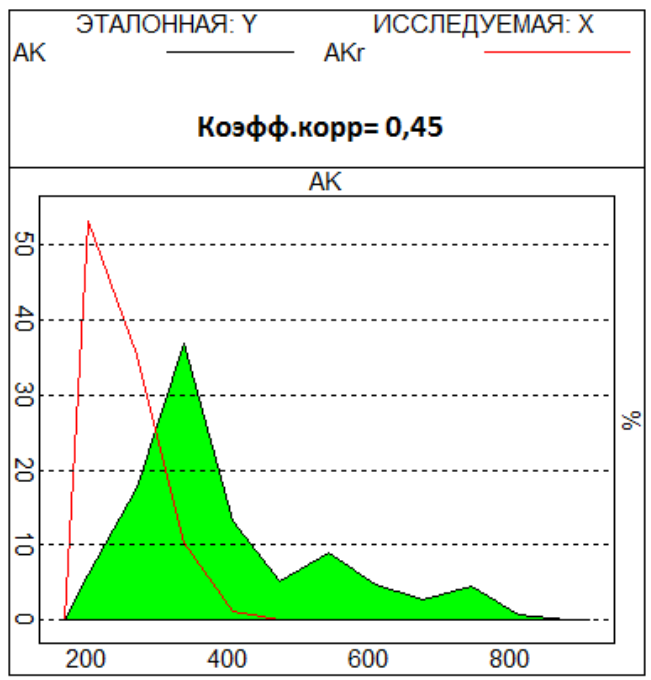

(4)

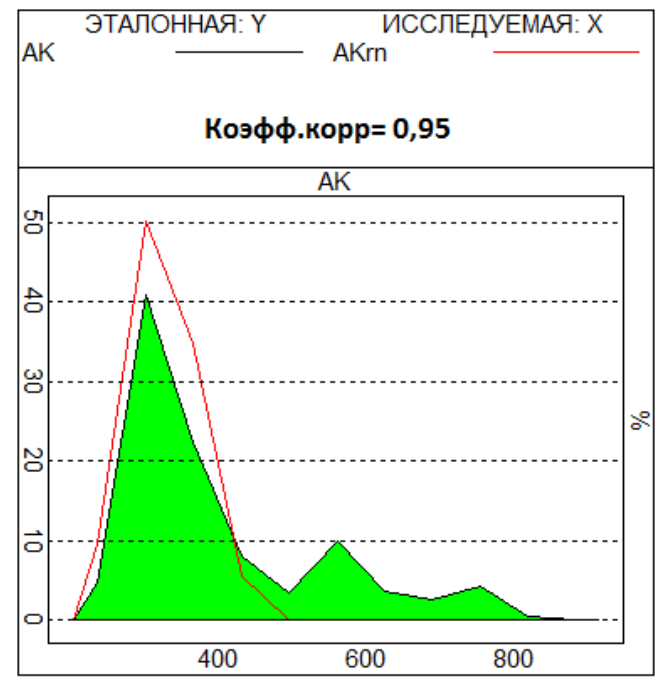

Рисунок 3 Стандартизачия кривой АК. Коэффищиент коррелящии до и после нормировки

В процессе обработки каротажных кривых АК и ГГК-П происходит уменьшение ошибок Ркав, $\mathrm{Pa}, \mathrm{Pн,} \mathrm{в} \mathrm{результате} \mathrm{чего} \mathrm{возрастает} \mathrm{их} \mathrm{качество} \mathrm{-} \mathrm{КК.}$ 
В качестве формального показателя эффективности обработки можно рассматривать показатель:

$$
\mathrm{K} \ni \mathrm{o}=\frac{\mathrm{KKes}-\mathrm{KKKex}}{1-\mathrm{KKex}}
$$

где Кэо - показатель эффективности обработки, ККвх - значение показателя качества кривой до обработки, ККвых - значение показателя качества после обработки.

Поскольку предложенный показатель качества кривых является интегральным, в дополнение к нему предлагается использовать функцию надежности кривых:

$$
\mathrm{KH}(\mathrm{z})=1-\frac{\Phi 1(\mathrm{z})+\Phi 2(\mathrm{z})+\Phi 3(\mathrm{z})}{3},
$$

где Ф1, Ф2, Ф3 - перечисленные выше факторы (влияние каверн, аппаратурные ошибки, не стандартизованные кривые), принимающие значения 0 (отсутствует фактор), 1 (присутствует фактор), z - глубина.

При $\mathrm{KH}(\mathrm{z})=1$, влияние перечисленных факторов отсутствует.

При $\mathrm{KH}(\mathrm{z})=0$, все перечисленные факторы присутствуют (это наименее надежные участки кривых ГИС).

Функция надежности может рассчитываться до и после обработки кривых ГИС. При этом, если влияние какого либо фактора уверенно исключено, то он принимается равным 0 при расчетах, в противном случае - 1 .

При построении сейсмогеологической модели функция надежности, играя роль протокола состояния данных, дает дифференциальную (поинтервальную, поточечную) оценку субъективности кривых ГИС и указывает места, к которым нужно в первую очередь вернуться при рассогласовании комплекса данных ГИС - сейсморазведка.

На этапе комплексирования данных ГИС и сейсморазведки, обладая такой дополнительной информацией, специалистам легче принять решение, связанное с оценкой качества результирующей сейсмогеологической модели и корректировкой, при необходимости, графа обработки входной информации.

Во второй части доклада рассматривается проблема частичного или полного отсутствия записи в скважинах необходимых методов ГИС. Рассмотрены различные методики восстановления акустических и плотностных свойств разреза по данным ГИС.

На основе анализа опыта использования известных и авторских методик предлагается методическая схема выбора и реализации наиболее эффективных способов восстановления акустических и плотностных параметров в скважинах (рисунок 4).

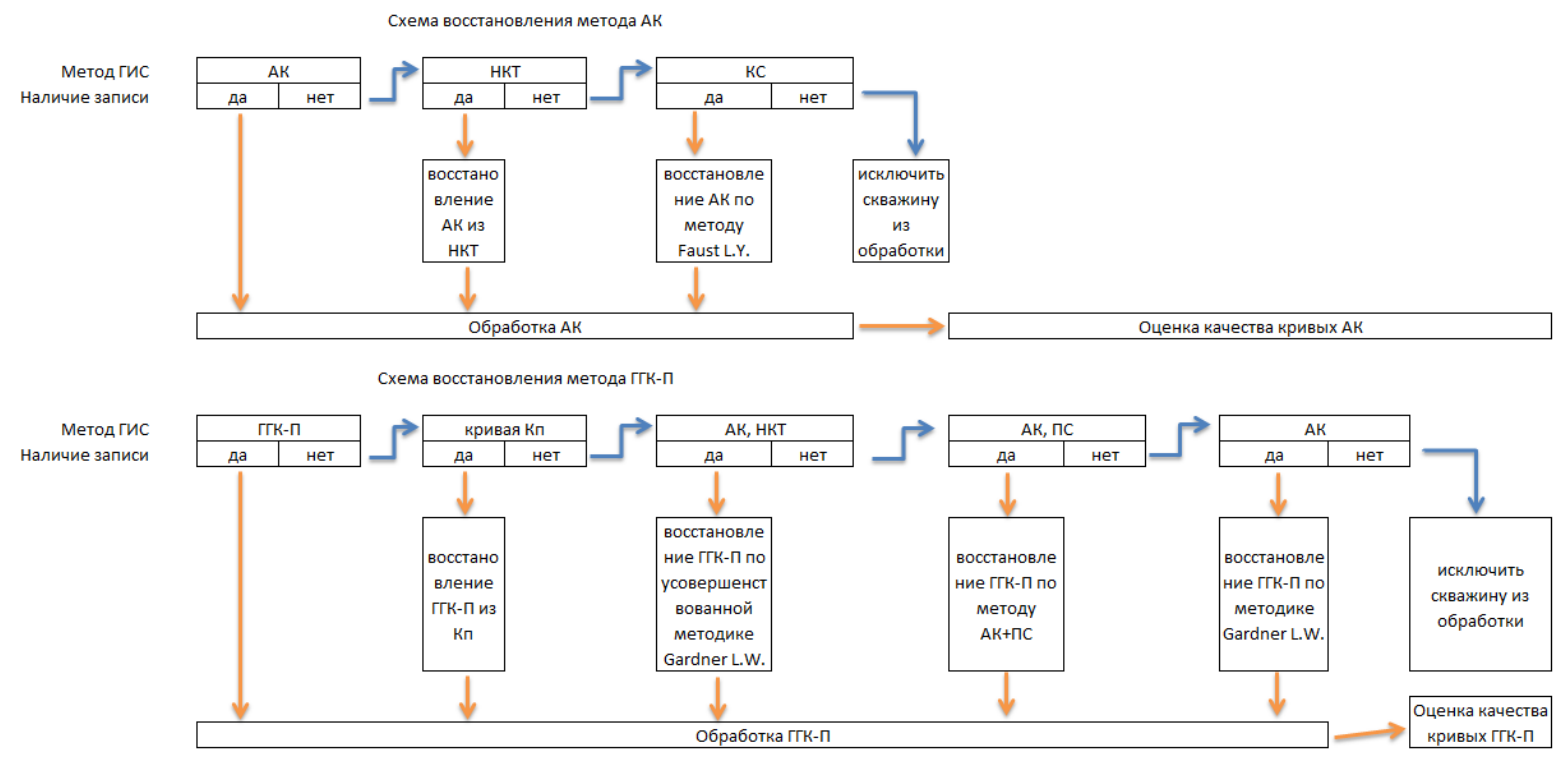

Рисунок 4 Методическая схема восстановления кривых АК и ГГК-П по данным ГИС 
Используя различные методики, мы получаем восстановленные параметры различного качества. Принципиально важным является количественная оценка качества восстановленных и измеренных параметров, используемых при построении сейсмогеологической модели. Развивая подход, предложенный в первой части доклада для интегральной оценки качества восстановленных кривых АК и ГГК-П по интервалу обработки, предлагается показатель качества - ККв, количественно учитывающий показатели качества входных данных и выбранного метода восстановления кривых.

$K_{\mathrm{B}}=\mathrm{PB}_{\mathrm{B}} \cdot \mathrm{P}_{\mathrm{M}}$,

где ККв - коэффициент качества восстановленных данных, Рв - показатель качества входных данных, Рм - показатель качества метода восстановления.

Качество входных данных (НКТ, КС, АК), используемых при моделировании, можно оценить путем их приведения к эталонным величинам опорной скважины. Показателем качества входных данных (Рв) в данном случае будет величина коэффициента корреляции между значениями входных кривых (НКТ, КС, АК) стандартизируемой и опорной скважин. Для оценки качества (Рм) выбранного метода восстановления кривых необходимо по предлагаемым методикам сопоставить в эталонной скважине зарегистрированные и синтетические (восстановленные) кривые АК и ГГК-П. Коэффициент корреляции зарегистрированных и синтетических кривых будет характеризовать качество метода восстановления.

\section{Выводы}

Целесообразно развитие предлагаемого подхода на другие этапы обработки и интерпретации данных ГИС и сейсмогеологического моделирования, совершенствование методического и алгоритмического обеспечения настройки предлагаемого подхода применительно к конкретным (типовым) сейсмогеологическим условиям.

Предлагаемая формализация процесса обработки позволяет управлять указанным процессом, сделать его более прозрачным, объективным, контролируемым. Кроме того, такая формализация позволяет формировать базы данных и знаний для эффективного решения задач геологического моделирования по комплексу данных ГИС-сейсморазведка.

Также формализация методик позволяет повысить эффективность подготовки и повышения квалификации специалистов, то есть перевести эту деятельность из разряда искусства в разряд наукоемких технологий. Это особенно важно в многоэтапных процессах геологического моделирования.

\section{Благодарности}

За помощь и поддержку выражаю искренне признание научному руководителю, доктору технических наук, профессору С.К. Туренко; Тюменскому индустриальному университету и Филиалу «ЛУКОЙЛ-Инжиниринг» «КогалымНИПИнефть» в г. Тюмени за возможность проведения исследований и опробования их на материалах компании. Также хочу поблагодарить коллег: Г.В. Такканда за ценные советы по теме работы, Я.А. Калачеву и Ю.А. Теплых за помощь в опробовании предложенных методик.

\section{Библиография}

1. Черепанов Е.А. К оценке качества результатов обработки данных ГИС с позиции построения сейсмогеологических моделей. / Е.А. Черепанов, С.К. Туренко // Сборник докладов совещаниясеминара. - Тюмень, 2015. - С. 43-47.

\section{References}

1. Cherepanov E.A. and Turenko S. K., 2015, On the quality assessment of LOG data processing results from the position of seism geological models construction: Tyumen, Collection of reports of a meeting seminar, p. 43-47 